\title{
The Uveal Effusion Syndrome and Trans-Scleral Flow
}

\author{
J. V. FORRESTER, ${ }^{*}$ W. R. LEE, P. R. KERR, H. S. DUA* \\ Aberdeen and Glasgow
}

\begin{abstract}
Summary
Recent studies of the uveal effusion syndrome, a rare condition characterised by idiopathic spontaneous serous detachment of the retina and peripheral choroid, have suggested a primary scleral abnormality as the underlying cause. In particular, abnormal deposition of glycosaminoglycans within the sclera may impair normal trans-scleral flow of fluid and contribute to increased scleral thickness. In four cases of uveal effusion syndrome, we have confirmed the accumulation of glycosaminoglycans in the sclera. Histochemical studies show that most of this material is proteodermatan sulphate with a smaller contribution from proteochondroitin sulphate, while electron microscopy showed an increase in collagen fibril thickness. Secondary changes within the retinal pigment epithelium were also observed, particularly foci of proliferation which corresponded to the characteristic 'leopard-spot' fundal appearances of this disorder. We therefore suggest that the uveal effusion syndrome is due to a primary defect in proteodermatan synthesis and/or degradation by scleral fibroblasts and may represent a form of ocular mucopolysaccharidosis.
\end{abstract}

Spontaneous serous detachment of the choroid was described clinically by von Graefe in $1858^{1}$ and histologically by Verhoeff and Waite. ${ }^{2}$ This rare condition is characterised by serous detachment of the peripheral choroid and ciliary body in an annular fashion with bullous, shifting non-rhegmatogenous retinal detachment. Both eyes are usually affected, sometimes with a time interval in onset, and in some series males predominate. ${ }^{3}$ Patients may present when vision deteriorates due to involvement of the macula in the exudative detachment. ${ }^{4}$ Gass and Jallow ${ }^{4}$ described several other characteristic features including the leopard-spot pigment epithelial changes, the consistent ultrasonic evidence of choroidal thickening and of peripheral choroidal and ciliary body detachment.

Idiopathic serous detachment of the choroid and retina is usually considered clinically, and probably also pathogenetically, distinct from serous detachments in nanophthalmic eyes, where the eye is small and there is chronic hypotony. ${ }^{5}$ The pathogenesis of idiopathic serous detachment is obscure. On clinical evidence of marked episcleral venous distension, it has been suggested ${ }^{6}$ that fluid accumulation may be due to impairment of venous drainage secondary to the extensive scleral thickening previously observed. ${ }^{3}$ Gass and Jallow ${ }^{4}$ observed that Schlemm's canal frequently filled with blood indicating elevated back venous episcleral pressure, and fluorescein angiography has confirmed that choroidal perfusion (flow) is significantly delayed. ${ }^{6}$ Several other conditions which may be associated with impaired venous drainage, are characterised by choroidal detachments including compressive and/or inflammatory diseases of the orbit and globe (e.g. scleritis,

From: Department of Ophthalmology, University of Aberdeen* and Department of Pathology, University of Glasgow.

Correspondence to: Professor J. V. Forrester, Department of Ophthalmology, Aberdeen University Medical School, Foresterhill, Aberdeen AB9 2ZD. 
Table I Uveal effusion syndrome: clinical data

\begin{tabular}{|c|c|c|c|c|c|}
\hline & Age & Sex & Presentation & Laterality & Outcome \\
\hline Case 1 & 65 & F & Visual loss, progressive inferior RD? solid & Unilateral & Enucleation \\
\hline Case 2 & 64 & $\mathbf{M}$ & Visual loss, rapid inferior RD? solid & Unilateral & Enucleation \\
\hline Case 3 & 56 & $\mathbf{M}$ & Visual loss, gradual total RD? annular melanoma & Unilateral & Enucleation \\
\hline Case 4 & 63 & $\mathbf{M}$ & Visual loss, gradual inferior RD pigmentation & Bilateral & $\begin{array}{l}\text { Scleral } \\
\text { resection }\end{array}$ \\
\hline
\end{tabular}

scleral buckling procedures), vortex vein anomalies and pan-retinal photocoagulation (for review see ref 7). On this basis, therefore, treatment of spontaneous uveal effusion by decompression of the vortex veins has been attempted $^{89}$ with varying success.

Gass $^{10}$ also found that simple lamellar resection of the sclera produced as good a result as the more difficult operation of vortex vein decompression. He hypothesised that protein-rich choroidal and sub-retinal fluid accumulated because of a scleral abnormality,

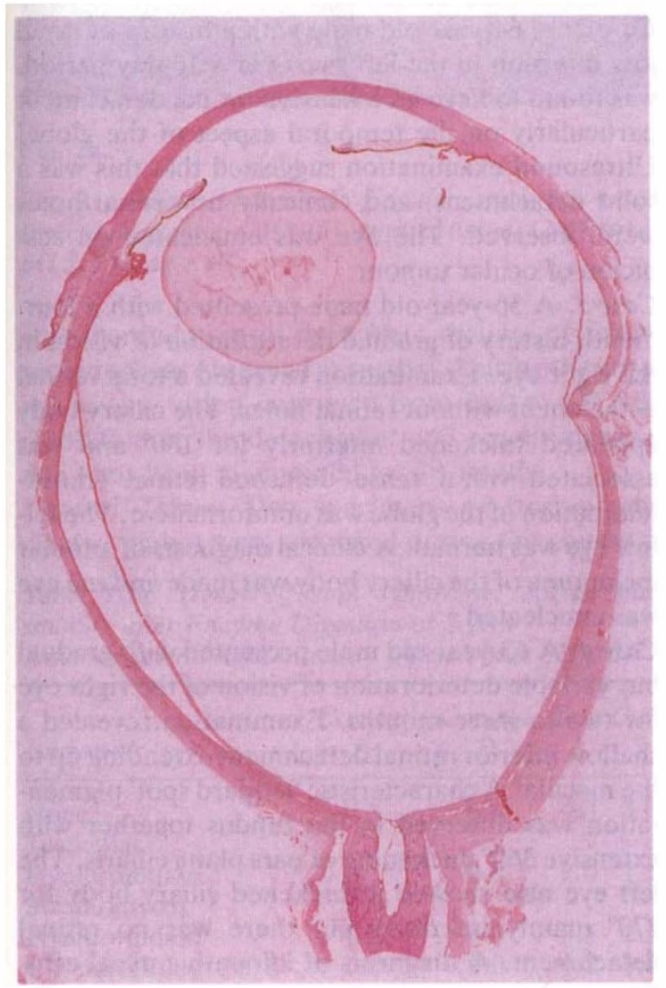

Fig. 1 a. characterised by the thickened sclera. A similar observation was noted in a rare case of uveal effusion syndrome associated with Hunter's syndrome, ${ }^{11}$ in which there are abnormal deposits of glycosaminoglycans in the tissues, including the sclera. More recently, increased quantities of scleral glycosaminoglycans were demonstrated by light and electron microscopy in a case of idiopathic uveal effusion symdrome. ${ }^{12}$

If Gass is correct, and there is compelling circumstantial evidence to support him, then

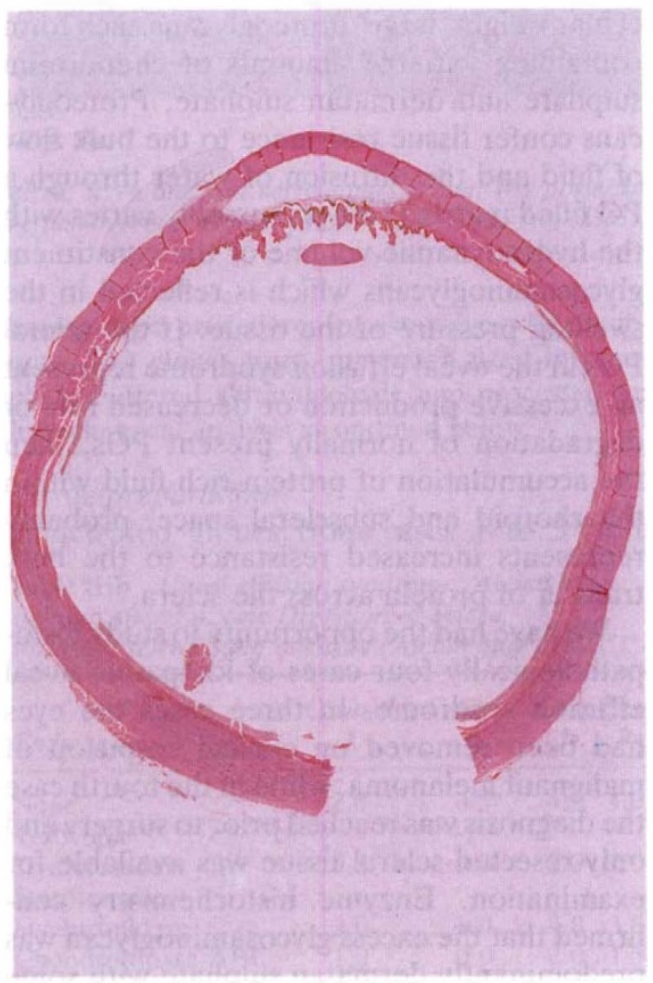

Fig. 1 .

Fig. 1. Sections of whole eyes stained with periodic acid Schiff reagent. (a) control eye (exenteration specimen); (b) uveal effusion eye (case 3). Note gross oedema of uvea in (b) with increased scleral thickness and intensity of stain in sclera. Control specimen shows artificial detachment of retina (Mag $\times 1.5)$. 
Table II Uveal effusion syndrome, Alcian blue staining Scleral stain intensity*

\begin{tabular}{lllllll}
\hline & \multicolumn{3}{c}{ Anterior } & \multicolumn{3}{c}{ Posterior } \\
\multicolumn{4}{c}{$p H 0.4 p H 1.0 p H 2.5$} & $p H 0.4 p H 1.0 p H 2.5$ \\
\hline Control A & 0.5 & 0.5 & 0.5 & 1.0 & 0.5 & 2.0 \\
Control B & 0.5 & 1.0 & 0.5 & 0.5 & 0.5 & 0.5 \\
\hline Case 1 & 2.0 & 2.0 & 4.0 & 2.0 & 1.0 & 4.0 \\
Case 2 & 2.0 & 2.5 & 3.0 & 2.0 & 3.0 & 3.0 \\
Case 3 & 2.0 & 2.5 & 3.0 & 3.0 & 3.0 & 3.0 \\
Case 3 & 2.5 & 3.0 & 2.5 & - & - & - \\
\hline
\end{tabular}

${ }^{*} 0=$ nil $; 1=$ minmal $; 2=$ mild $; 3=$ moderate $; 4=$ marked.

it would be important to identify the glycosaminoglycans or more accurately the proteoglycans, which are present in excess in the sclera in the uveal effusion syndrome. Normal sclera contains low to moderate amounts of proteoglycans (PGs) as either low molecular weight, 'small' proteoglycans, or high molecular weight 'large' proteoglycans each form containing variable amounts of chondroitin sulphate and dermatan sulphate. Proteoglycans confer tissue resistance to the bulk flow of fluid and the diffusion of water through a PG filled matrix. This presumably varies with the hydrodynamic volume of the constituent glycosaminoglycans which is reflected in the swelling pressure of the tissue. If the scleral PGs in the uveal effusion syndrome represent an excessive production or decreased rate of degradation of normally present PGs, then the accumulation of protein-rich fluid within the choroid and subscleral space, probably represents increased resistance to the bulk transfer of protein across the sclera.

We have had the opportunity to study histopathologically four cases of idiopathic uveal effusion syndrome. In three cases the eyes had been removed on clinical suspicion of malignant melanoma, while in the fourth case the diagnosis was reached prior to surgery and only resected scleral tissue was available for examination. Enzyme histochemistry confirmed that the excess glycosaminoglycan was predominantly dermatan sulphate with some increase in chondroitin sulphate. Electronmicroscopy also confirmed the abnormal scleral collagen changes. In addition, abnormalities were also observed in the ret- inal pigment epithelial cell layers, which may have been secondary to the long standing retinal detachment, but which correlate clinically with the 'leopard-spot' fundal changes.

\section{Materials and Methods}

\section{Cases}

Ocular tissue from four cases of idiopathic uveal effusion syndrome have been received by the Department of Pathology, University of Glasgow during the period 1977-1987 (Table I). In three cases, the entire globe was enucleated due to clinical suspicion of ocular melanoma. In the furth case, strips of sclera were obtained after a scleral resection procedure.

Case 1: A 65-year-old female with a history of several weeks progressive loss of upper nasal visual field loss, was found to have a lower temporal, clinically 'solid' retinal detachment. The fellow eye was normal. The eye was enucleated on suspicion of a choroidal melanoma.

Case 2: A 64-year-old male with a history of rapid loss of vision in the left eye over a 10-day period, was found to have an extensive retinal detachment particularly on the temporal aspect of the globe. Ultrasound examination suggested that this was a solid detachment, and clinically no retinal holes were observed. The eye was enucleated on suspicion of ocular tumour.

Case 3: A 56-year-old male presented with a fourmonth history of gradual deterioration of vision in the right eye. Examination revealed a total retinal detachment without retinal holes. The ciliary body appeared thickened inferiorly for $180^{\circ}$ and was associated with a 'tense' detached retina. Transillumination of the globe was uninformative. The fellow eye was normal. A clinical diagnosis of annular melanoma of the ciliary body was made and the eye was enucleated.

Case 4: A 63-year-old male presented with gradual but variable deterioration of vision of the right eye for two to three months. Examination revealed a shallow inferior retinal detachment extending up to the macula. A characteristic 'leopard spot' pigmentation was observed in the fundus together with extensive $360^{\circ}$ thickening of pars plana ciliaris. The left eye also showed a thickened ciliary body for $270^{\circ}$ mainly inferiorly but there was no retinal detachment. A diagnosis of idiopathic uveal effusion syndrome was made.

A course of systemic steroids had no effect on the subretinal exudate and scleral resection in four quadrants were performed, as described by Gass ${ }^{9}$ but without sclerotomy or sclerostomy. Consider- 


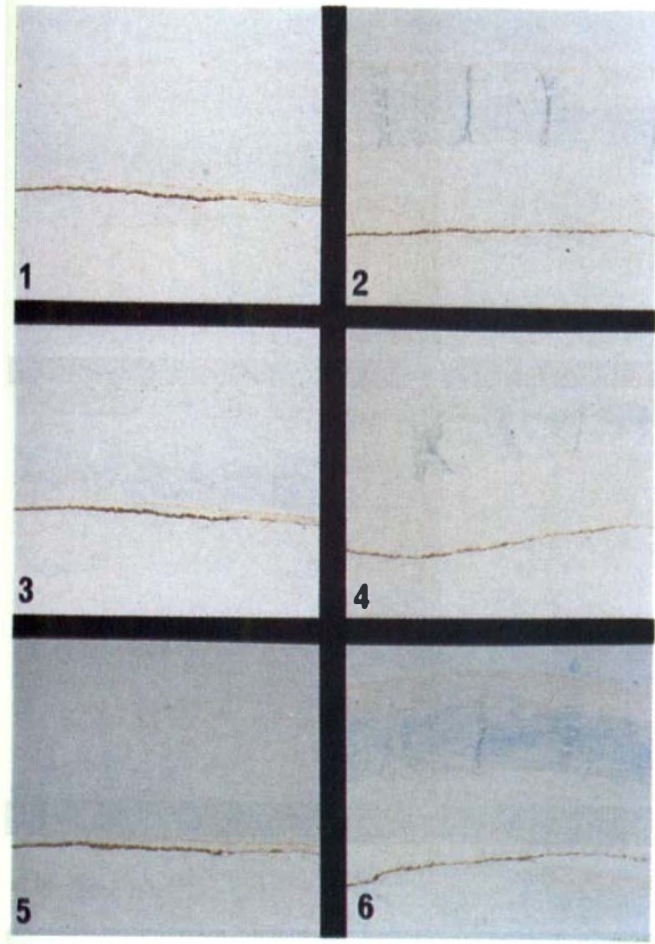

Fig. 2a.

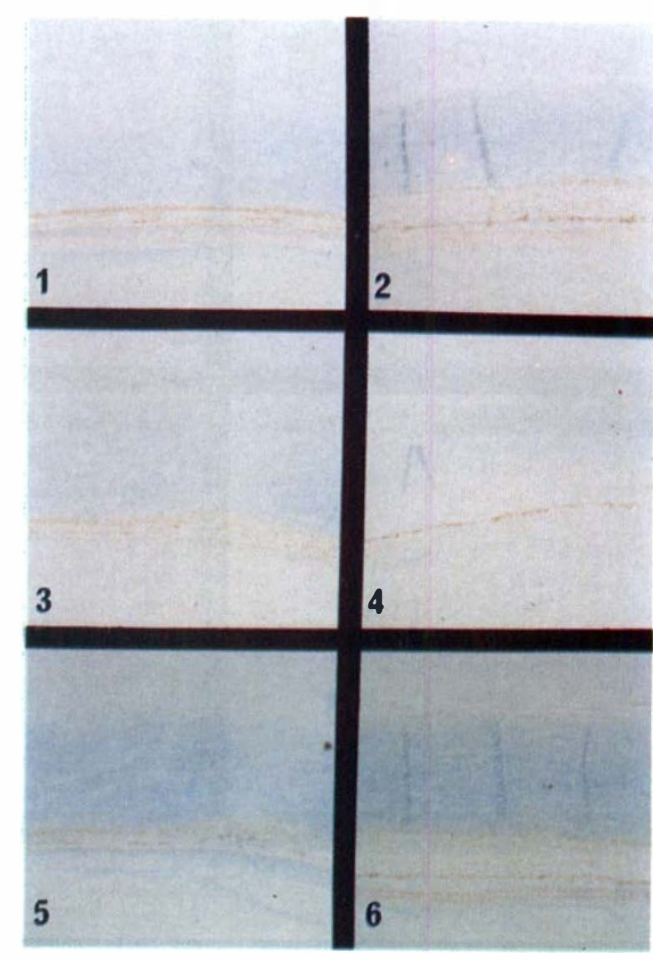

Fig. 2b.

Fig. 2. Alcian blue stain of ocular coats. (a) anterior sclera, uvea and ora serratalpars plana; (b) posterior (macular) region. (1)(3)(5), control tissue; (2)(4)(6), uveal effusion tissue. (1) (2), pH 0.4; (3) (4), pH 1.0; (5) (6), pH 2.5 (Mag $\times 40)$.

able transudation of fluid from the site of scleral resection was observed at surgery. During the ensuing weeks, vision improved from $6 / 60$ to $6 / 9$ with resolution of the detachment and visual improvement has been maintained for 18 months.

Control Tissue: Two specimens of freshly enucleated globes were obtained during the course of

Table IIIa Uveal effusion syndrome, Alcian blue staining after Enzyme Digestion of Sclera

Anterior scleral stain intensity (Alcian blue pH 2.5)*

\begin{tabular}{lcccc}
\hline Enzyme & $\begin{array}{c}\text { Control } \\
A\end{array}$ & $\begin{array}{c}\text { Control } \\
\text { None }\end{array}$ & \multicolumn{2}{c}{ Case Case } \\
\hline Nesticular & 0.5 & 0.5 & 4.0 & 3.0 \\
Hyaluronidase & 0 & 0.0 & 2.0 & 1.5 \\
Streptomyces & & & & \\
Hyaluronidase & 0.5 & 1.0 & 3.5 & 2.5 \\
Chondroitinase ABC & 0.0 & 0.0 & 1.5 & 1.0 \\
Chondroitinase AC & 0.5 & 0.5 & 3.5 & 3.0 \\
Neuraminidase & 1.0 & 0.5 & 4.0 & 4.0 \\
\hline
\end{tabular}

*0 0 nil $1=$ minimal $; 2=$ mild $; 3=$ moderate $; 4=$ marked. exenteration procedures for extensive orbital tumours. The globes were immersion fixed in phosphate buffered glutaraldehyde and processed for histochemical analysis as outlined below.

\section{Tissue preparation}

Enucleated globes from cases 1 to 3 were

Table IIIb Uveal effusion syndrome, Alcian blue staining after Enzyme Digestion of Sclera

Posterior scleral stain intensity (Alcian blue pH 2.5)*

\begin{tabular}{lcccc}
\hline & Control & \multicolumn{4}{c}{ Control } & \multicolumn{2}{c}{ Case Case } \\
Enzyme & $A$ & $B$ & 1 & 3 \\
\hline None & 2.0 & 0.5 & 4.0 & 3.0 \\
Testicular & & & & \\
Hyaluronidase & 0.5 & 0.0 & 2.0 & 2.0 \\
Streptomyces & & & & \\
Hyaluronidase & 3.0 & 1.0 & 4.0 & 3.0 \\
Chondroitinase ABC & 1.0 & 0.0 & 2.0 & 1.5 \\
Chondroitinase AC & 1.0 & 0.5 & 4.0 & 3.0 \\
Neuraminidase & 3.0 & 0.5 & 4.0 & 4.0 \\
\hline
\end{tabular}

* $0=$ nil $; 1=$ minimal $2=$ mild $; 3=$ moderate $; 4=$ marked. 


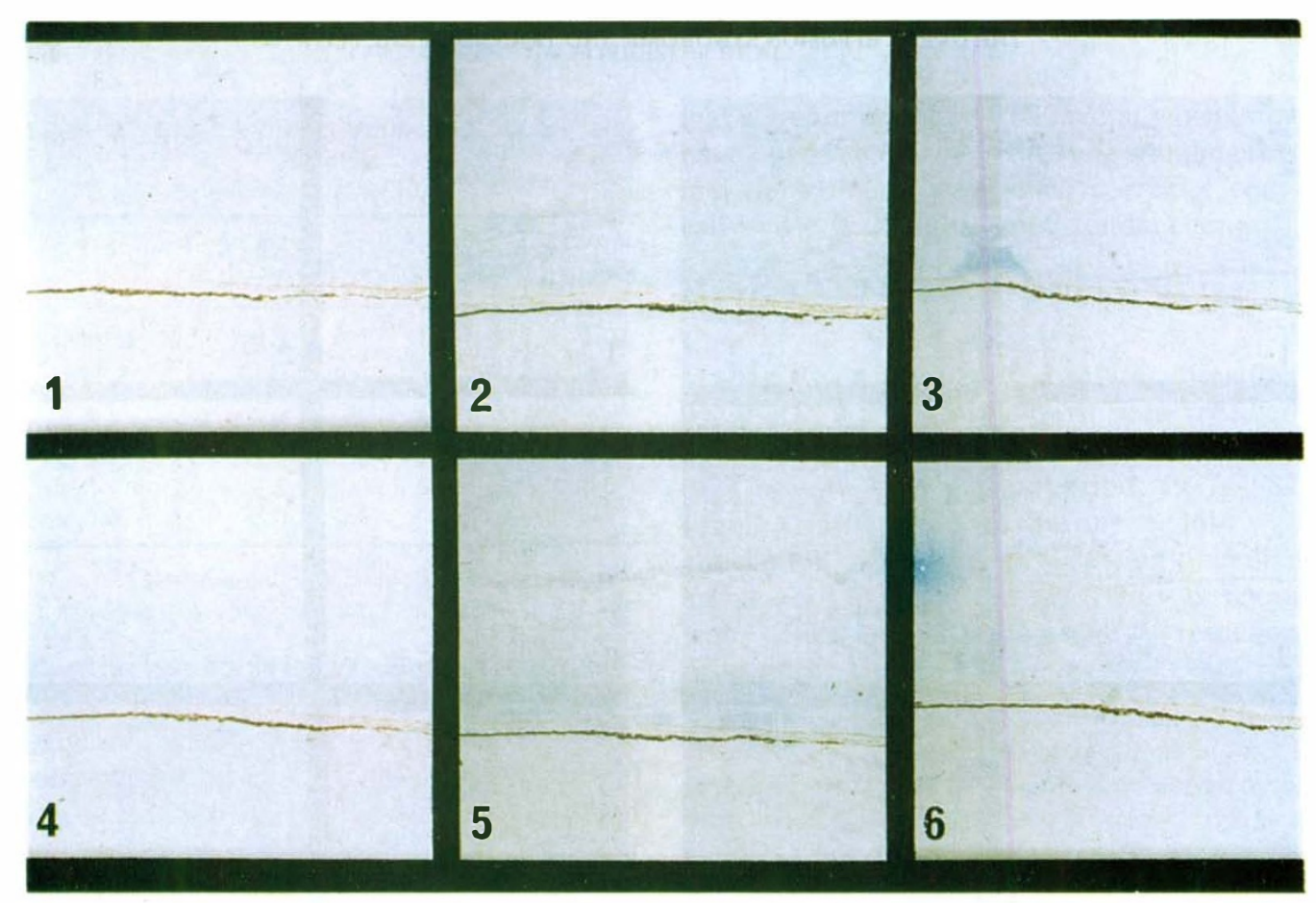

Fig. 3a.

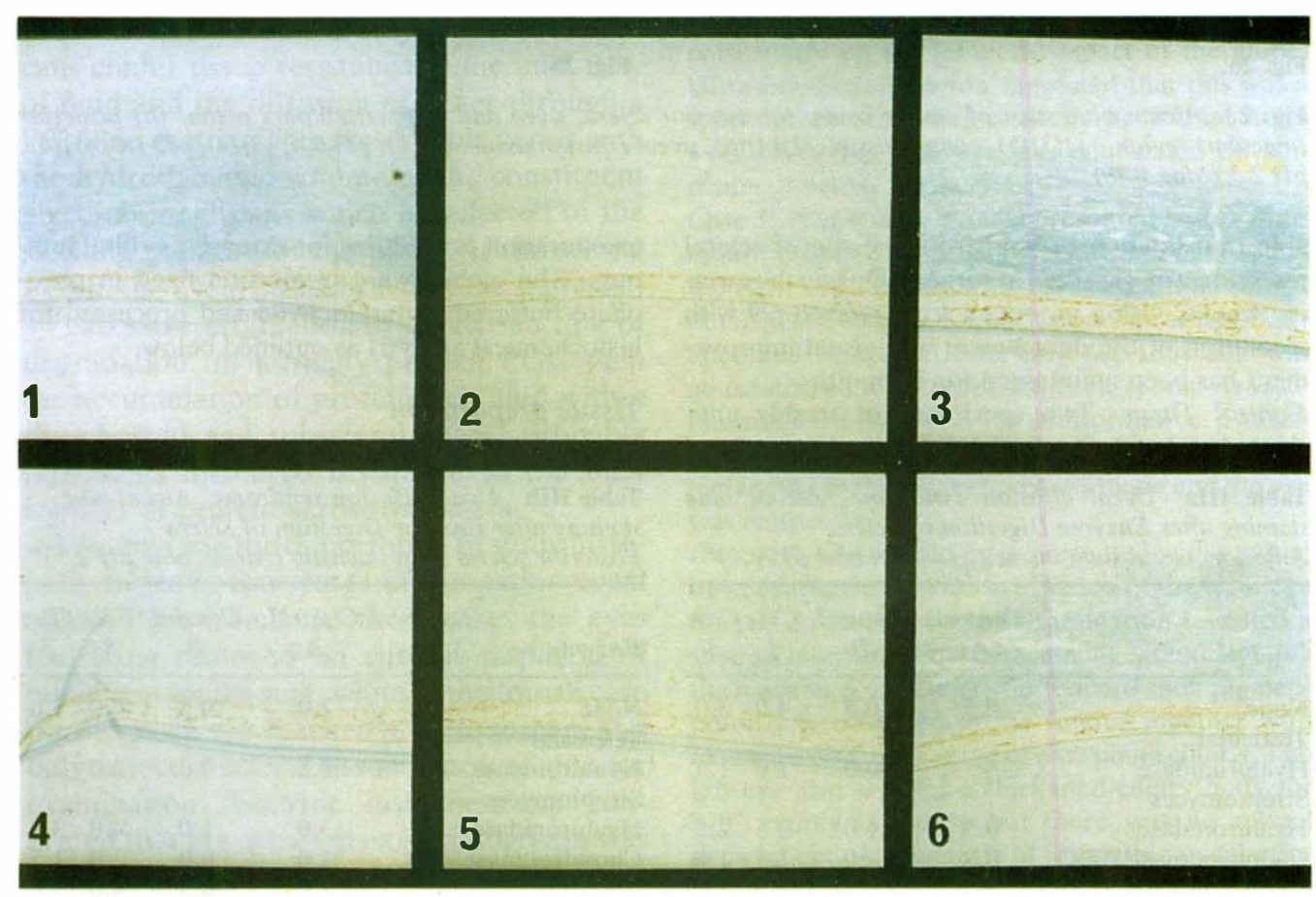

Fig. 3b.

Fig. 3. Enzyme histochemistry of normal eye post-stained with Alcian blue, pH 2.5. (a) anterior segment; (b) posterior segment. Sections were pretreated with (1) no enzyme (2) testicular hyaluronidase (3) streptomyces hyaluronidase (4) chondroitinase $A B C$ (5) chondroitinase $A C$ (6) neuraminidase. Note difference in staining intensity between anterior sclera $(a, 1)$ and posterior sclera $(b, 1)$. Note preferential reduction in stain with testicular hyaluronidase $(a, 2 ; b, 2)$ and chondroitinase $A B C(a, 4 ; b, 4)($ Mag $\times 40)$. 


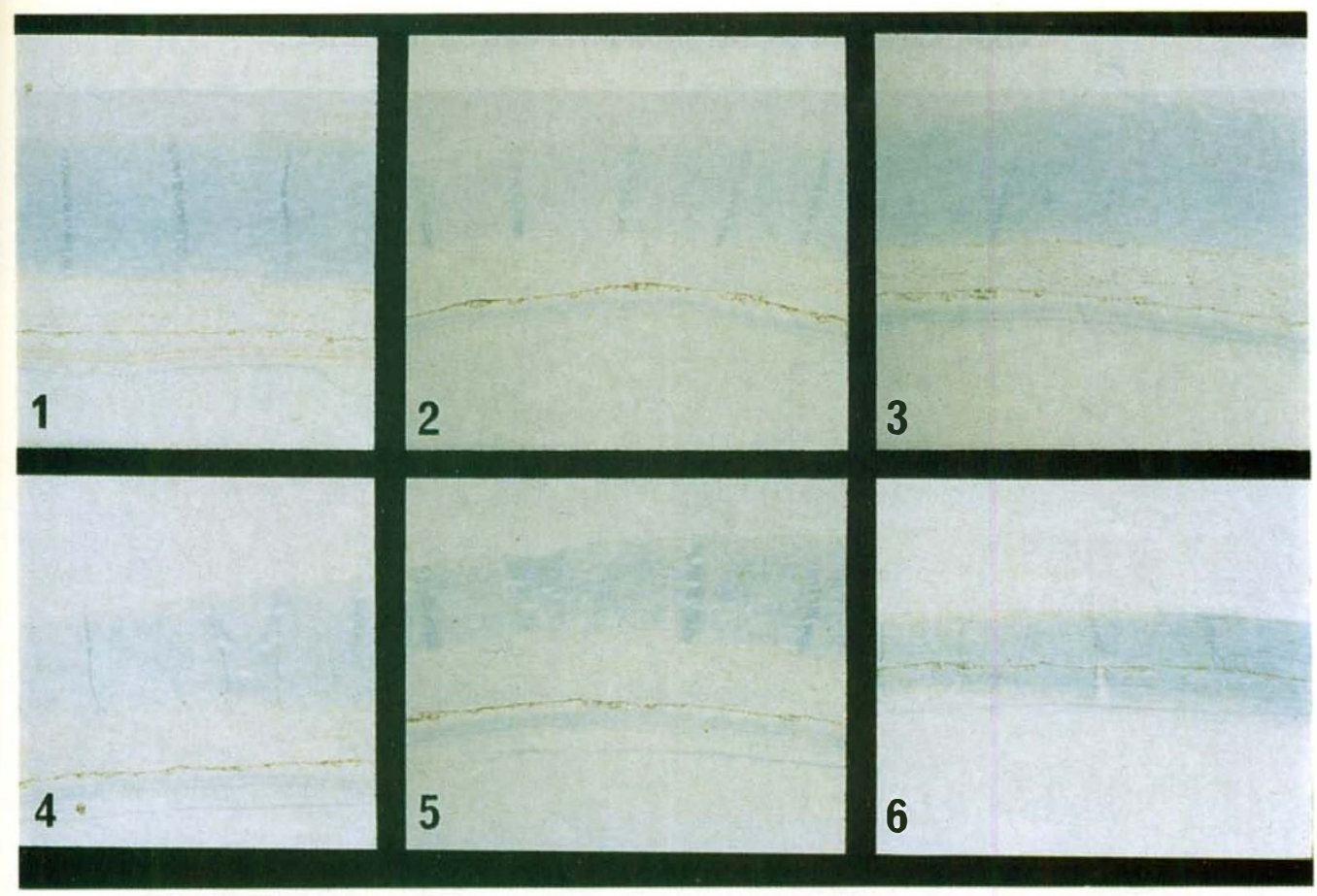

Fig. 4a.

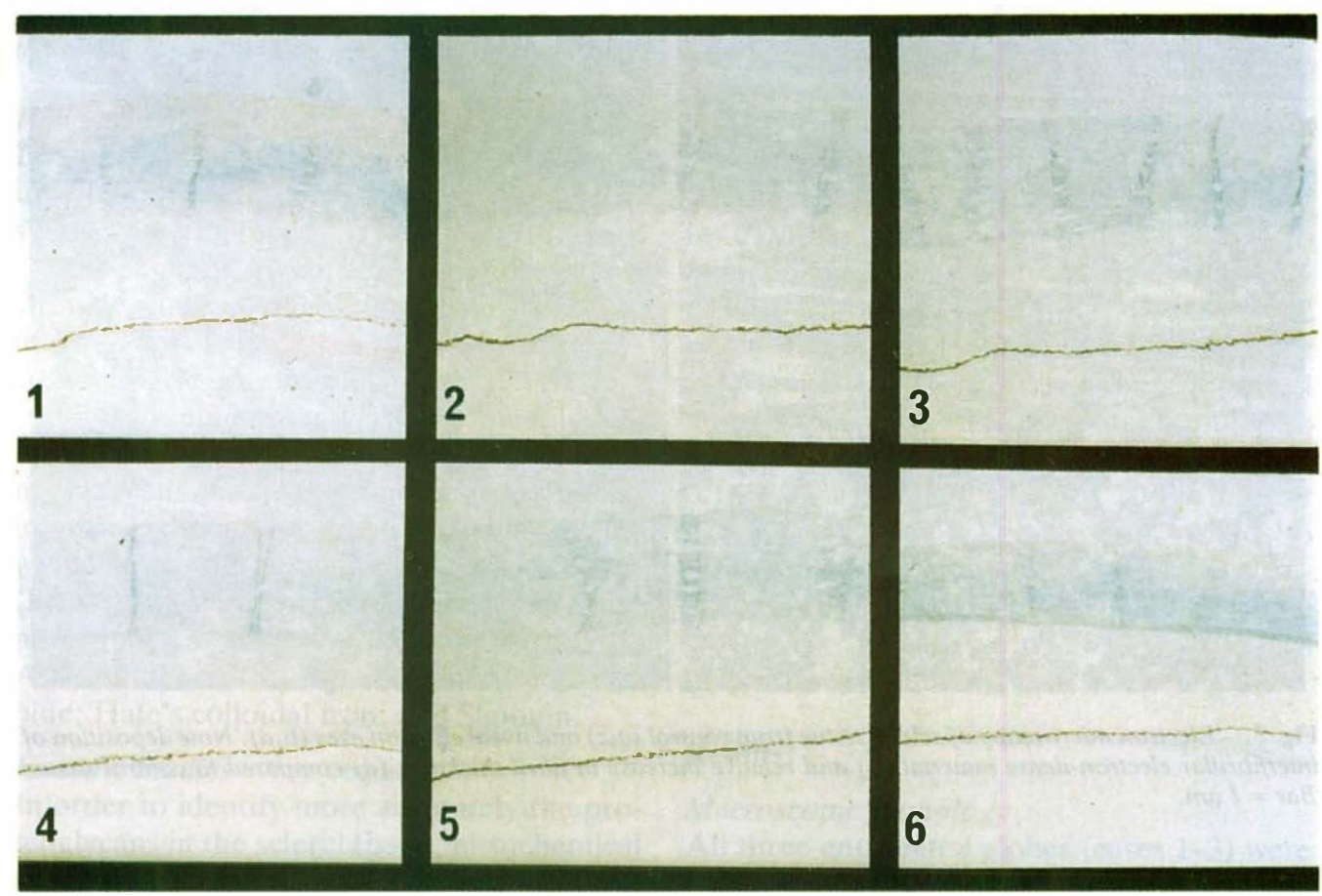

Fig. 4b.

Fig. 4. Enzyme histochemistry of uveal effusion eye post-stained with Alcian blue, pH 2.5. (a) anterior segment, (b) posterior segment. Sections were pretreated with (1) no enzyme (2) testicular hyaluronidase (3) streptomyces hyaluronidase (4) chondroitinase ABC (5) chondroitinase AC (6) neuraminidase. Note reduction in stain with testicular hyaluronidase $(a, 2 ; b, 2)$ and chondroitinase $A B C(a, 4 ; b, 4)(M a g \times 40)$. 


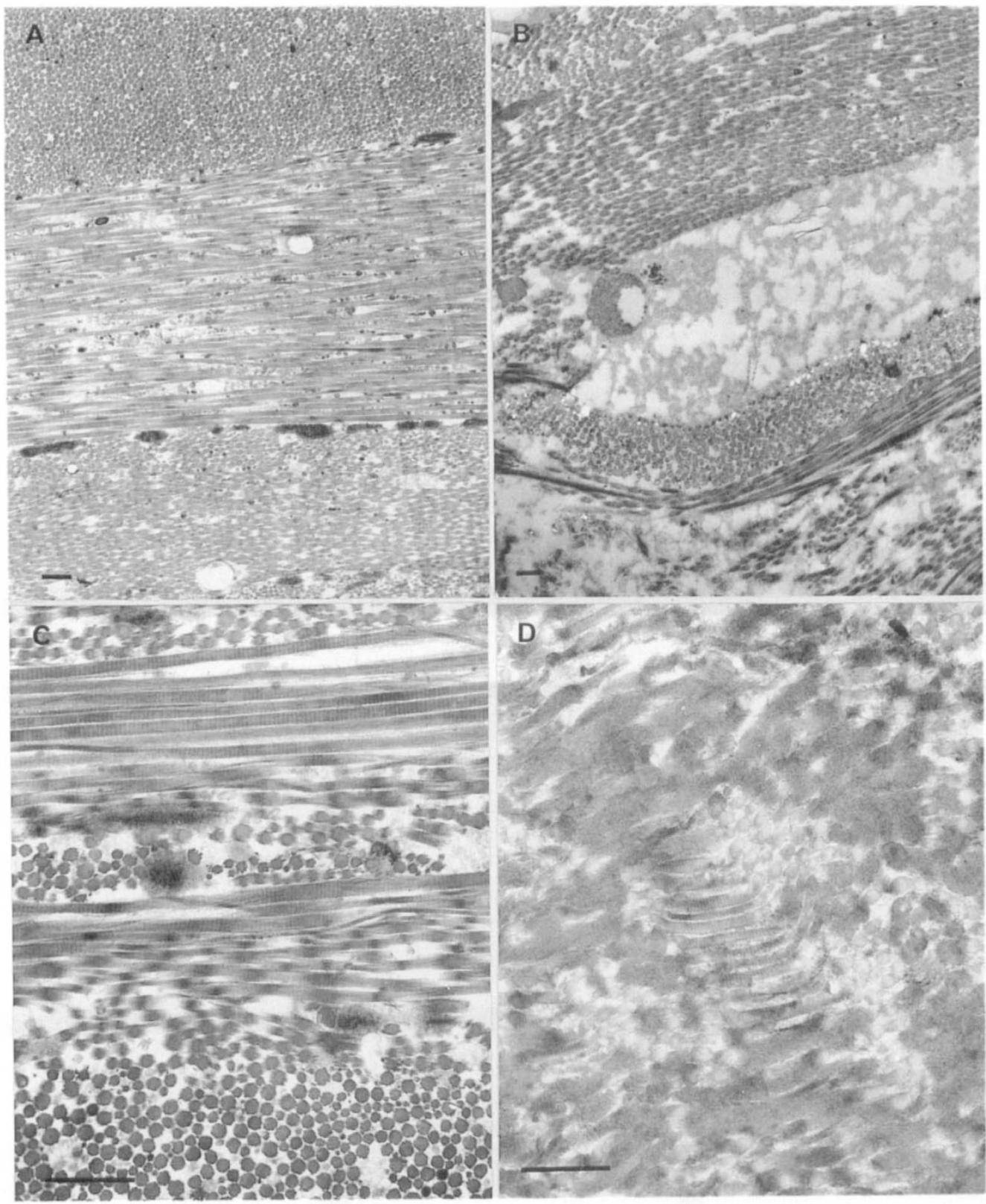

Fig. 5. Electron microscopy of scleral tissue from control $(a, c)$ and uveal effusion eyes $(b, d)$. Note deposition of interfibrillar electron-dense material (b) and relative increase in fibril thickness $(d)$ compared to control tissue. Bar $=1 \mu \mathrm{m}$.

immersed in cacodylate-buffered gluteraldehyde and processed by conventional means for light and electron microscopy. Resected scleral tissue from Case 4 ( $4 \times 4$ mm blocks) was similarly prepared for light and electron microscopy. 


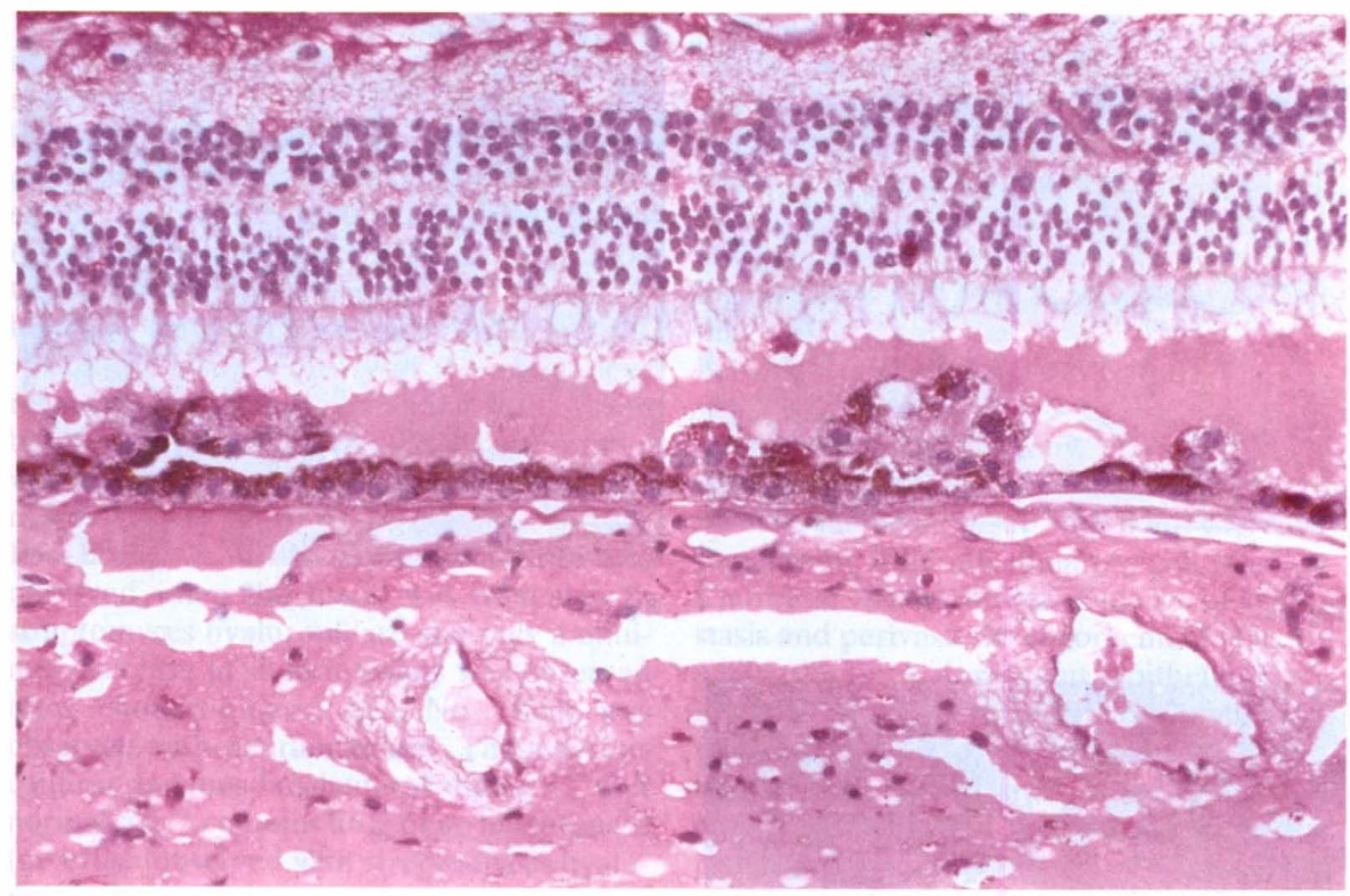

Fig. 6. Histological section of PAS stained uveal effusion tissue. Note dilated engorged choroidal veins, PAS positive inclusion in RPE layer and migration and proliferation of RPE cells in the subretinal space $($ Mag $\times 250)$.

\section{Histochemical stains}

In addition to haematoxylin and eosin staining for light microscopy, the following special stains were employed:

One per cent Alcian blue at pH 0.4 (stains strongly sulphated glycosaminoglycans and mucin) at pH 1.0 (stains weakly sulphated glycosaminoglycans and mucin), and at $\mathrm{pH} 2.5$ (stains all mucins and glycosaminoglycans including hyaluronic acid, sulphated glycosaminoglycans and neuraminidase-resistant sialomucin). In addition the following stains were employed: $1 \%$ Periodic Acid Schiff (PAS); PAS with periodate borohydride and potassium hydroxide, PB $(\mathrm{KOH})$ PAS; Alcian blue-PAS; High iron diamine Alcian blue; Hale's colloidal iron; and Saponin.

\section{Enzyme Studies}

In order to identify more accurately the proteoglycans in the scleral tissue, histochemical staining with $1 \%$ alcian blue, $\mathrm{pH} 2.5$ was combined with prior incubation of the tissue sections with the following enzymes: bovine testicular hyaluronidase, 100 units per $\mathrm{ml}$ in $0.1 \mathrm{M}$ phosphate buffer $\mathrm{pH} 5.5$ for two hours at $37^{\circ} \mathrm{C}$ (digests hyaluronic acid, chondroitin -4 and -6 sulphate and dermatan sulphate); streptomyces hyaluronidase, 100 units per $\mathrm{ml}$ in $0.1 \mathrm{M}$ phosphate buffer at $\mathrm{pH} 5.0$ for two hours at $60^{\circ} \mathrm{C}$ (specifically degrades only hyaluronic acid); chondroitinase $\mathrm{ABC}, 0.5-0.2$ units per $\mathrm{ml}$ in $0.1 \mathrm{M}$ Tris $\mathrm{HCl}$ at $\mathrm{pH} 8.0$ for 20-40 mins at $37^{\circ} \mathrm{C}$ (degrades chondroitin -4 and -6 sulphate and dermatan sulphate); chondroitinase $\mathrm{AC}, 0.5-0.2$ units per $\mathrm{ml}$ in $0.1 \mathrm{M}$ Tris $\mathrm{HCl}$ at $\mathrm{pH} 7.3$ for $20-40$ mins at $37^{\circ} \mathrm{C}$ (degrades only chondroitin -4 and -6 sulphate, but not dermatan sulphate); neuraminidase, $0.5 \mathrm{mg}$ per $\mathrm{ml}$ in $0.1 \mathrm{M}$ phosphate buffer for 18 hours at $37^{\circ} \mathrm{C}$ at $\mathrm{pH} 5.8$ (degrades sialomucin).

\section{Results}

\section{Macroscopic Pathology}

All three enucleated globes (cases 1-3) were within the range of normal dimensions. The retinae were detached and there was an extensive proteinaceous subretinal exudate, particularly in cases 1 and 3 . The sclera appeared uniformly thickened in case 3 , and 


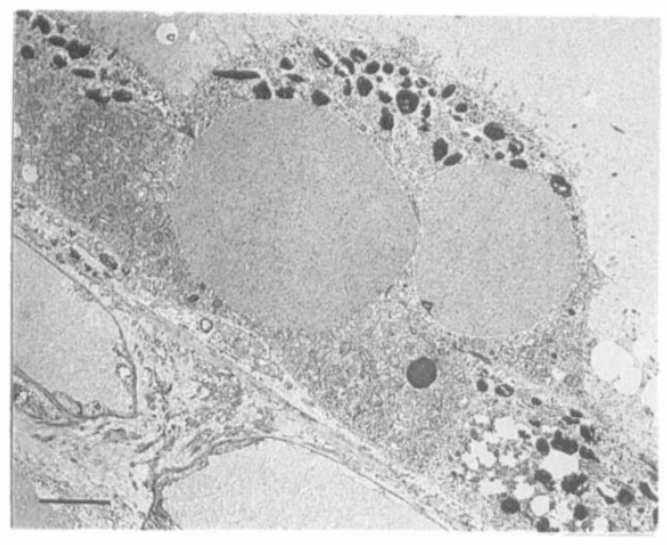

Fig. 7a.

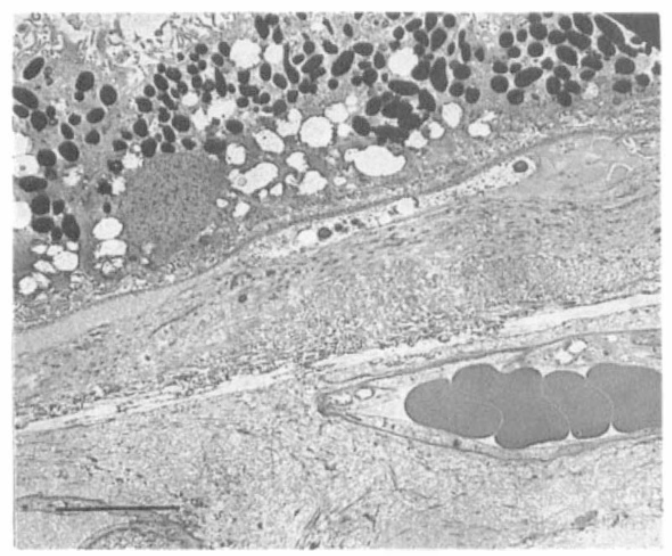

Fig. 7c.

thickened at the posterior pole in case 2, while it was of normal thickness in case 1 . Resected scleral tissue from the pre-equatorial sclera in case 4 was approximately $1.5 \times$ thickness of normal sclera. In cases $1-3$, there was obvious exudation in the ciliary body and anterior suprachoroidal space.

\section{Microscopic Pathology}

\section{(A) Scleral Pathology}

Increased quantities of PAS positive material in the sclera were observed in all four cases of uveal effusion syndrome when compared to the control tissue (Fig. 1). This was particularly marked in the post-macular scleral tissue. Strong staining was also observed with Hale's colloidal iron and high iron diamine Alcian blue, suggesting an

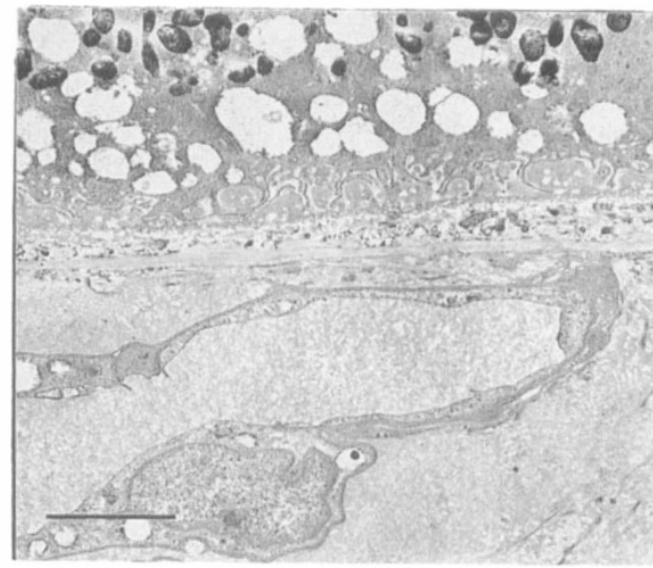

Fig. 7 b.

Fig. 7. Electron microscop! of retino-choroidal interface in areal effusion svindrome. (a) RPE Eells with single large prestein-rich inclusion; bar $=10 \mu \mathrm{m}$, (b) Basal infoldings in RPE (e'ls; bar $=5 \mu \mathrm{m}$. (c) Thickening of Bruch's membrane below RPE cells with deposition of electron dense material; har $=5 \mu \mathrm{m}$.

excessive deposition of sulphated mucins in the uveal cffusion cases.

Alcian blue staining of the sclera at different $\mathrm{pH}$ levels revealed some interesting findings. In the normal eye there was generally weak Alcian blue staining but there was a marked difference in staining between the pre-equatorial (anterior) sclera and the paramacular (posterior) sclera particularly at $\mathrm{pH}$ 2.5. This suggests that in the normal eye sulphated proteoglycans are uniformly distributed throughout the sclera, but that non-sulphated glycosaminoglycans (such as hyaluronate) and sialomucins may be concentrated in the para-macular sclera. In addition, most of the Alcian blue-positive material was located in the inner sclera, while the outer sclera and episclera were negative. 
In eyes with uveal effusion, there was a marked increase in Alcian blue positive material (Table II; Fig. 2a, b) at all pH levels in both anterior and posterior sclera. This suggests that in addition to increased deposits of strong and weakly sulphated proteoglycans (detected at $\mathrm{pH} 0.4$ and 1.0 respectively) additional non-sulphated proteoglycans and sialomucins may have been present.

Enzyme histochemistry of the normal eye revealed a reduction in staining with Alcian blue at $\mathrm{pH} 2.5$ when the tissue was treated with testicular hyaluronidase and chondroitinase $A B C$ as described in Methods (Table IIIa, b; Fig. 3a, b). No reduction in stain was observed when the tissue was treated with streptomyces hyaluronidase and only a minimal decrease in stain intensity was observed after chondroitinase AC. No effect was observed with neuraminidase. These results confirm previous biochemical studies ${ }^{13,14}$ that normal sclera contains little or no hyaluronate (no effect observed with streptomyces hyaluronidase) and only a small proportion of total glycosaminoglycan is comprised of chondroitin -4 or -6 sulphate (partial effect with chondroitinase AC), whereas the major component is dermatan sulphate (removed by chondroitinase $\mathrm{ABC}$ and also removed by broad-spectrum testicular hyaluronidase). The anterior sclera contains little sialic acid, but the para-macular sclera may contain some neuraminidase-resistant sialic acid.

In the scleral tissue from uveal effusion syndrome eyes, there was a similar reduction in Alcian blue staining after testicular hyaluronidase and chondroitinase ABC treatment, but there was also a significant reduction in stain intensity after chondroitinase AC treatment (Table IIIa, b; Fig. 4a, b). Complete removal of Alcian blue reactivity was not achieved with the present enzyme treatments but this may have been a simple dose-saturation effect. These results indicated that in the uveal effusion syndrome, dermatan sulphate and chondroitin sulphate preferentially accumulated in the sclera, while no apparent accumulation of hyaluronate (resistant to streptomyces hyaluronidase) or sialomucins (no effect with neuraminidase) was observed.

Electron microscopy of scleral tissue from uveal effusion cases showed disruption of the normal lamellar arrangement of the collagen fibrils, numerous electron dense deposits in the interfibrillar spaces, and a greater variability in collagen fibril diameter with a general increase in fibril width compared to normal sclera (Fig. 5a, b). Sclera in case 4 showed clumped electron dense material between collagen fibres which had an indistinct cross-banding. Finely granular material was present between collagen fibrils in the control tissue.

\section{(B) Uveoretinal Pathology}

Despite the unusually massive engorgement of the uveal tissue with fluid, there was remarkably little inflammatory cell reaction. Choroidal vessels showed evidence of venous stasis and perivascular engorgement (Fig. 6), while the retinal pigment epithelium was hypertrophic and hyperplastic in foci beneath the detached retina. Many RPE cells contained large PAS positive inclusion bodies. Numerous RPE cell rests were engaged in proliferation and migration into the subretinal space (Fig. 6) which contained very large amounts of a highly proteinaceous fluid. The photoreceptor layer showed atrophic outer segment changes with vacuolation and cell loss, while the retina showed generalised minor atrophic changes.

By electron microscopy, the RPE cells showed signs of multilayering and migratory activity while their cytoplasm was frequently engorged with single circular protein-rich inclusions (Fig. 7a, b). Loss of transport function in the RPE cells was suggested by the abnormalities in basal infoldings (Fig. 7b) and stunting and loss of apical microvilli. However, tight junctions remained intact. Macrophages containing large inspissated proteinaceous inclusion bodies were also identified.

Bruch's membrane was considerably thickened and dilated with interfibrillar ground substances and electron dense round bodies (Fig. 7c). The choroid contained a massive proteinaceous exudate in the extravascular compartment.

\section{Discussion}

This review of four cases of uveal effusion syndrome has led to the following observations: 
(a) three of the four cases showed some degree of scleral thickness;

(b) the increase in scleral thickness was due to accumulation of glycosaminoglycan (proteoglycan) and also, in part, related to a variable increase in collagen fibril width;

(c) the increase in glycosaminoglycan content was mainly due to deposition of dermatan sulphate, and also, but less so, to chondroitin sulphate as revealed by enzyme digest studies;

(d) the engorged uveal tissue showed remarkably little inflammatory change;

(e) the retinal pigment epithelium showed marked changes, including foci of proliferation.

The results therefore confirm, and extend, the observation by Ward et al. that the abnormality in scleral tissue in the uveal effusion syndrome is characterised by abnormal deposits of glycosaminoglycan and which are now shown to be predominantly dermatan sulphate. Gass ${ }^{9}$ further hypothesised that the pathogenesis of the uveal effusion syndrome was related to the abnormal deposits of glycosaminoglycans since they retarded the transcleral diffusion or flow of protein-rich fluid. In addition, due to their highly hydrophilic nature the increased glycosaminoglycans bind increased quantities of water, which cause an increase in the intrascleral swelling pressure and inhibit venous drainage through the vortex veins, thereby causing further uveal engorgement.

Gass' hypothesis implies therefore that idiopathic uveal effusion syndrome represents a primary defect in scleral fibroblast production of proteoglycans. Recent biochemical and electron microscopical studies, including some very elegant rotary shadowing ${ }^{15}$ and $\mathrm{X}$-ray diffraction ${ }^{16}$ experiments, have studied the relationship between proteoglycans and collagen fibrils in sclera. Both dermatan sulphate and chondroitin sulphate are disaccharide polymers of $\mathrm{N}$-acetyl-galactosamine and either of the epimers, iduronic acid (dermatan sulphate) and glucuronic acid (chondroitin sulphate). Chondroitin sulphate contains no iduronic acid, and in the tissues it is only loosely associated with the collagen fibrils, essentially lacking the space between groups of fibrils. Dermatan sulphate, in contrast, contains varying amounts of iduronic acid from $95 \%$ to $10 \%$, the remainder of the disaccharides in the polymer containing glucuronic acid. ${ }^{15}$ Both dermatan sulphate and chondroitin sulphate exist in the tissues bound to a core protein as proteoglycans. Dermatan sulphate proteoglycan occurs as two forms, 'small' proteodermatan (PGI) containing large quantities of iduronic acid (up to $95 \%$ ) and 'large' proteodermatan (PGII) with about $10 \%$ iduronic acid. ${ }^{17}$ PGII co-exists with chondroitin sulphate proteoglycan within the interfibrillar space. In contrast, a 'small' PGI combines specifically with the ' $\mathrm{d}, \mathrm{e}$ ' region of the banded collagen fibril in a regular pattern, and completely encases the fibril. ${ }^{16,18}$ Scott and Hughes ${ }^{19}$ have shown that PGI is instrumental in regulating collagen fibril width or thicknesś during development, and may be important in determining whether Type I collagens form part of an interstitial matrix as in the dermis or whether they become specialised to form specific collagen structures of high tensile strength such as occur in tendons and sclera.

The presence of increased quantities of dermatan sulphate in the uveal effusion syndrome may therefore have some influence on collagen fibril diameter which may account for the variable width and apparent increased thickness of collagen fibril observed in this study (Fig. 5a, b).

As noted above, these observations suggest an intrinsic defect in scleral fibroblast metabolism since these cells are the most likely source of sclera proteoglycans. An alternative possibility, however, is that the primary defect lies in the retinal pigment epithelium since this layer is crucial to the active transport of fluid across the retina to the sclera. Considerable abnormalities were observed in the RPE layer in this study, including loss and stunting of apical microvilli, protein-rich intracellular inclusions, and morphologically altered basal infoldings. These findings might indicate a functional defect in the transport of fluid across the RPE but are more likely to be secondary to the longstanding retinal detachment, since similar findings may be observed in rhegmatogenous detachments. In addition, the protein-rich subretinal exudate probably acted as a stimulus for the increased phagocytic, proliferative and migratory activity of the RPE cells in uveal effusion syndrome. 
Clinically, the leopard-spot pigmentary fundal changes would correspond to the foci of RPE proliferations and multi-layering.

In conclusion, therefore, this study supports the proposal by Gass ${ }^{9}$ that the accumulation of scleral, choroidal and subretinal protein-rich fluid is probably due to abnormally thickened sclera; causing increased back-venous pressure with excessive transudation of fluid from the choroidal vasculature. The increased scleral thickness is due to an accumulation of proteoglycan, specifically dermatan sulphate proteoglycan with a smaller contribution from chondroitinsulphate proteoglycan. This further increases resistance to transcleral flow of protein-rich fluid, which increases the colloid osmotic pressure of the tissues. It is likely that the accumulation of scleral proteoglycan is a primary defect and may represent an ocular form of mucopolysaccharidosis, since similar findings have been observed in a case of the systemic mucolysaccharidosis, Hunter's syndrome. ${ }^{10}$

\section{References}

${ }^{1}$ von Graefe A: Zur diagnose des beginnenden intraocularen Krebses. Albrecht von Graefes Arch. fur Ophthalmologie 1858, 4: 2180-229.

${ }^{2}$ Verhoff FH and Waite JH: Separation of the choroid with report of a spontaneous case. Trans Am Ophthalmol Soc 1858, 23: 120-39.

${ }^{3}$ Schepens CL and Brockhurst RJ: Uveal Effusion 1. Clinical Picture. Arch Ophthalmol 1963, 70: 189-201.

${ }^{4}$ Gass JDM and Jallow S: Idiopathic serous detachment of the choroid, ciliary body and retina (uveal effusion syndrome). Ophthalmology 1982, 89: 1018-32.

${ }^{5}$ Brockhust RJ: Nanophthalmic eyes with uveal effusion. A new clinical entity. Arch Ophthalmol 1975, 93: 1289-99.
${ }^{6}$ Wilson RS and Hanna C: Idiopathic chorioretinal effusion: an analysis of extra-cellular fluids. Ann Ophthalmol 1977, 9: 647-53.

${ }^{7}$ Brubakerr RF and Pederson JE: Ciliochoroidal detachment. Surv Ophthalmol 1983, 27 : 281-89.

${ }^{8}$ Brockhurst RJ: Vortex vein decompression for nanophthalmic uveal effusion. Arch Ophthalmol 1980, 98: 1987-90.

${ }^{9}$ Casswell AG, Gregor ZJ, Bird AC: The surgical management of uveal effusion syndrome. Eye 1987, 1: 115-19.

${ }^{10}$ Gass JDM: Uveal effusion syndrome. A new hypothesis concerning pathogenesis and technique of surgical treatment. Retina 1983, 3: 159-63.

${ }^{11}$ Vine AK: Uveal effusion in Hunter's syndrome. Retina 1986, 6: 57-60.

${ }^{12}$ Ward RC, Gragondas ES, Pon DM, Albert DM: Abnormal scleral findings in the uval effusion syndrome. Am J Ophthalmol 1985, 106: 139-46.

${ }^{13}$ Coster L and Fransson L-A: Isolation and characterisation of dermatan sulphate proteoglycans from bovine sclera. Biochem J 1981, 193: 143-53.

${ }^{14}$ Coster L, Rosenberg LC, van der Rest M, Poole AR: The dermatan sulphate proteoglycans of bovine sclera and their relationship to articular cartilage. J Biol Chem 1986, 262: 3809-12.

${ }^{15}$ Ward MP, Scott JE, Coster L: Dermatan sulphate proteoglycans from sclera examined by rotary shadowing and electron microscopy. Biochem $J$ 1987, 242: 761-6.

${ }^{16}$ Quantock AJ and Meek KM: Axial electron density of human scleral collagen. Location of proteoglycans by X-ray diffraction. Biophys J 1988, 54: 159-64.

${ }^{17}$ Coster L, Rosenberg LC, van der Rest M, Poole AR: The dermatan sulphate proteoglycans of bovine sclera and their relationship to those of articular cartilage. J Biol Chem 1987, 262: 3809-812.

${ }^{18}$ Young RD: The ultrastructural organisation of proteoglycans and collagen in human and rabbit scleral matrix. J Cell Sci 1985, 74: 95-104.

${ }^{19}$ Scott JE and Hughes EW: Proteoglycan collagen relationships in developing chick and bovine tendons. Influence of the physiological environment. Con Tissue Res 1986, 14: 267-78. 